\title{
Use of the facial dismasking flap approach for surgical treatment of a multifocal craniofacial abscess
}

\author{
Yoshitaka Ishii ${ }^{1}$, Tomoyuki Yano ${ }^{2}$, Osamu Ito ${ }^{3}$ \\ ${ }^{1}$ Department of Plastic and Reconstructive Surgery, Shinmatsudo Central General Hospital, Matsudo; ${ }^{2}$ Department of Plastic and \\ Reconstructive Surgery, Cancer Institute Hospital, Tokyo; ${ }^{3}$ Department of Plastic and Reconstructive Surgery, Yokohama City Minato Red \\ Cross Hospital, Yokohama, Japan
}

The decision of which surgical approach to use for the treatment of a multifocal craniofacial abscess is still a controversial matter. A failure to control disease progress in the craniofacial region can potentially put the patient's life at risk. Therefore, understanding the various ways to approach the craniofacial region helps surgeons to obtain satisfactory results in such cases. In this report, we describe a patient who visited the emergency department with a large swelling in his right cheek. A blood test and computed tomography revealed odontogenic maxillary sinusitis. The patient developed sepsis due to a progressive multifocal abscess. An

Correspondence: Tomoyuki Yano Department of Plastic and Reconstructive Surgery, Cancer Institute Hospital, 3-8-31 Ariake, Koto-ku, Tokyo, Japan

Tel: +81-3-3520-0111

Fax: +81-3-3520-0141

E-mail:yanoaprs@icloud.com abscess was seen in the temporal muscle, infratemporal fossa, and interorbital region. To control this multifocal abscess, we used the facial dismasking flap (FDF) approach. After debridement using the FDF approach, we succeeded in obtaining sufficient drainage of the abscess, and the patient recovered from sepsis. The advantages of the FDF approach are that it provides a wide surgical field, extending from the parietal region to the mid-facial region, and that it leaves no aesthetically displeasing scars on the face. The FDF approach may be one of the best options to approach multifocal abscesses in the craniofacial region.

Keywords Face / Orbit / Abscess / Facial bones

Received: 31 May $2017 \bullet$ Revised: 22 Jul $2017 \bullet$ Accepted: 19 Sep 2017

pISSN: 2234-6163・ elSSN: 2234-6171 • https://doi.org/10.5999/aps.2017.00969・Arch Plast Surg 2018;45:271-274

\section{INTRODUCTION}

A multifocal craniofacial abscess is a rather rare condition, and no consensus exists regarding the best standardized surgical protocols for effective debridement of such abscesses. Performing sufficient and appropriate drainage of all abscesses is the basic principle of treatment in such cases. If a patient has a multifocal abscess in the craniofacial region, it is rather challenging to find an appropriate approach to access all foci of the abscess, because surgeons should consider not only controlling the patient's disease, but also the aesthetics and functional morbidity of the face. Multiple incisions and scars in the craniofacial region often have unfavorable aesthetic impacts on a patient, and pose a risk for the development of functional problems of the face, such as scar contracture, ptosis, ectropion, and facial palsy. Therefore, surgeons should carefully decide which approach to use when performing drainage of multifocal craniofacial abscesses.

In this report, we introduce the facial dismasking flap (FDF) approach as a treatment for a multifocal craniofacial abscess that led to minimal scarring in the face and no functional deficits resulting from the procedure. The FDF approach was originally designed and developed by Tajima et al. [1] to obtain complete 
exposure of the mid-face region without leaving an undesirable scar on the face or sacrificing the facial motor nerves. The FDF approach consists of a coronal incision with a hemilateral or bilateral circumpalpebral incision. After some modifications [2,3], this approach has been successfully used by surgeons to obtain a surgical field that is clear and wide enough to manipulate (Fig. 1), without leaving any undesirable scarring on the face or causing denervation of the facial motor nerves [4].

\section{CASE}

A 61-year-old male patient visited the emergency department of our hospital due to intense pain and swelling in his right cheek area. Diabetes was the only relevant comorbidity. A blood test

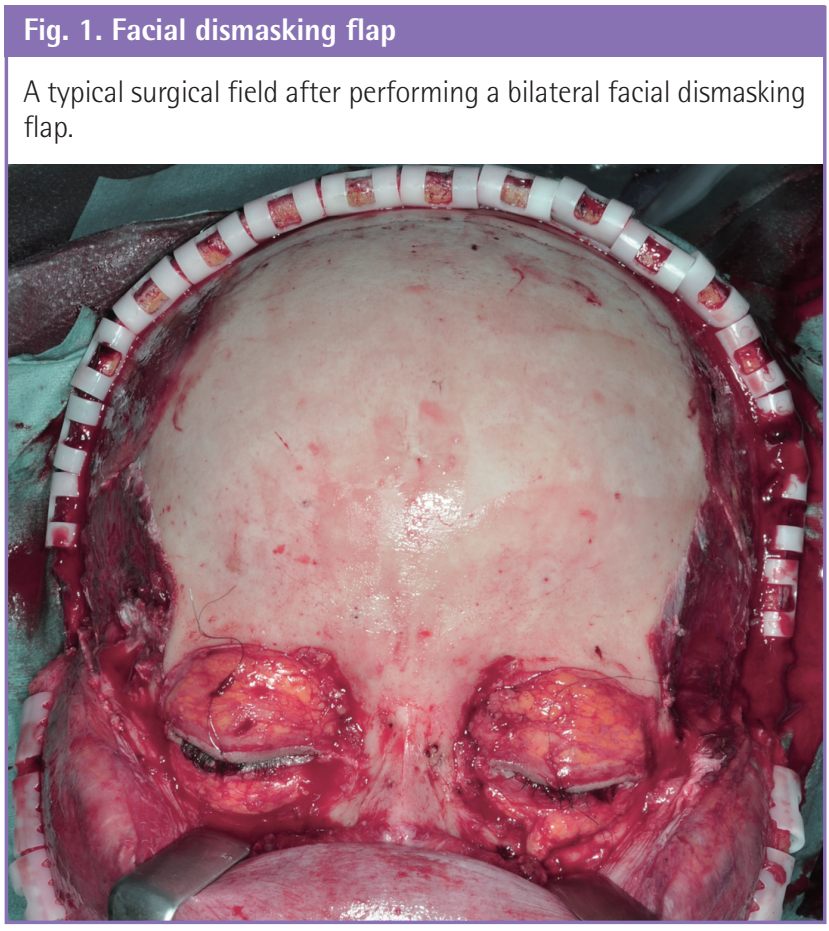

and computed tomography (CT) revealed odontogenic maxillary sinusitis accompanied by an abscess in the right lateral pterygoid muscle. The patient underwent immediate local intraoral debridement after admission to the hospital. However, the patient failed to recover and progressed into severe sepsis, with the abscess extending into the infratemporal fossa. The patient then underwent another radical debridement using a hemi-coronal incision and removal of a right upper tooth suffering from caries. Even after the second debridement, the patient remained in sepsis. Seven days after the first debridement, the patient suddenly developed a brain infarction and meningitis due to a thrombosis in the sigmoid sinus.

Furthermore, the patient had a recurrent abscess in the right infratemporal fossa (Fig. 2A) and another abscess in the right intraorbital region (Fig. 2B). The additional abscess in the intraorbital region seemed to pose the risk of spreading the infection into the intracranial region through the optic nerve and/or optic vessels (Fig. 2C). With these widespread multifocal abscesses in the craniofacial region, we decided to use the FDF approach to achieve thorough radical debridement.

The FDF approach was performed following the procedures described in the literature [2-5]. Through this approach, we obtained a surgical field that was clear and wide enough to observe and debride all abscesses in this patient. First, the right temporal muscle, which had already necrosed due to inflammation and infection of its feeding vessels, was excised (Fig. 3A). In the root of the temporal muscle, there was a recurrent abscess that had spread into the lateral pterygoid muscle, and this abscess was also debrided. Using the FDF approach, we were able to easily approach the right orbital wall. Part of the lateral orbital wall was removed in order to approach the intraorbital space using the FDF approach. With this additional lateral orbital approach incorporated into the FDF approach, we were able to reach the intraorbital abscess safely (Fig. 3B), and succeeded in draining

Fig. 2. Images of multiple abscesses

(A) Black arrows show an abscess in the inferior temporal fossa, (B) black arrows show abscesses in the intraorbital region, and (C) black arrows show abscesses along the superior rectus muscle deep into the orbital apex.
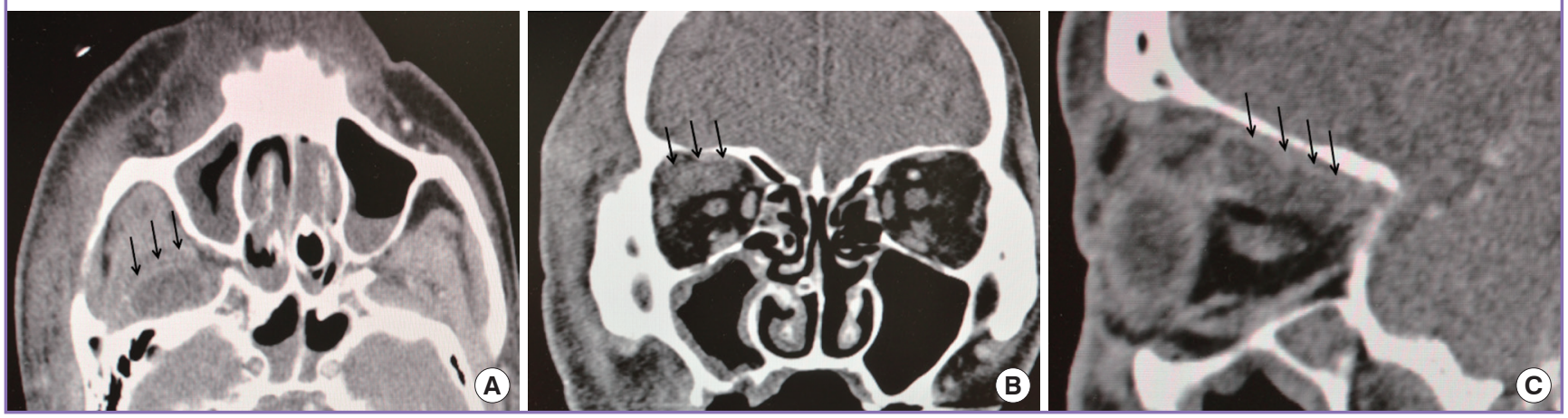
Fig. 3. Intraoperative images

(A) After performing the facial dismasking flap (FDF) approach, removal of the necrosed right temporal muscle and debridement of the right infratemporal fossa were performed. (B) Approach to the intraorbital abscess after removal of the right orbital wall combined with the FDF approach.
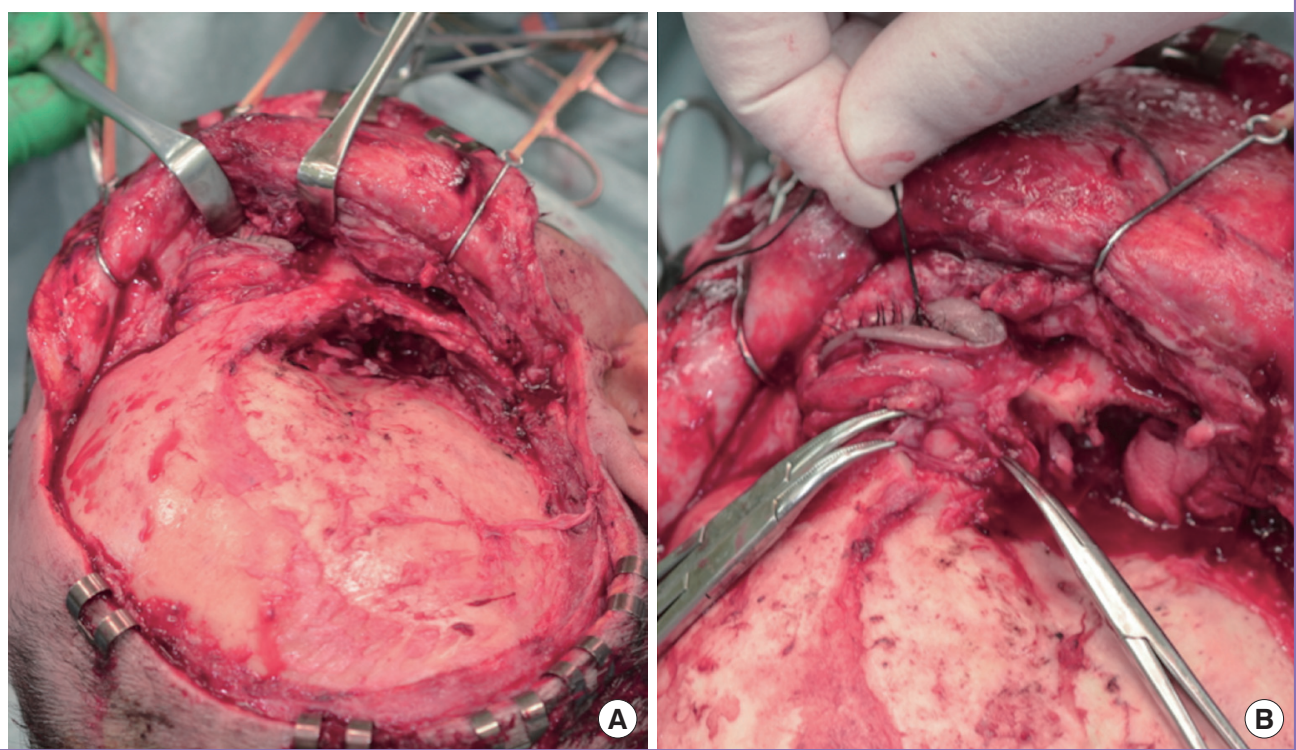

that abscess without causing contamination of the intracranial space. All wounds were irrigated with normal saline, and closed primarily in the ordinary fashion with the placement of Penrose drains.

After thorough debridement of the multifocal abscess using the FDF approach, the patient successfully recovered from severe sepsis. Postoperative $\mathrm{CT}$ images showed that no abscess was left in the craniofacial region; thus, we had achieved full control of the patient's disease and the patient recovered from sepsis. No unfavorable scarring occurred on the face and the facial nerves were not damaged, but there was a right temporal hollowing due to the excision of the right temporal muscle, as well as right ptosis due to cerebral infarction (Fig. 4).

\section{DISCUSSION}

The difficulty of controlling a multifocal craniofacial abscess depends on which surgical approach is chosen to achieve complete debridement. Without an appropriate approach to all affected areas, the disease will not be cured, as we experienced with our patient.

In our case, the approach to the intraorbital abscess was considered to be particularly difficult and a key point of treatment. It was difficult because there was a risk of damaging the optic nerve and/or vessels and contaminating the intracranial space if we failed to choose an appropriate approach for the intraorbital region. Moreover, if the patient underwent insufficient debridement, the disease would not be controlled.

Obtaining a wide surgical field to manipulate surgical instruments and perform sufficient debridement usually involves us-

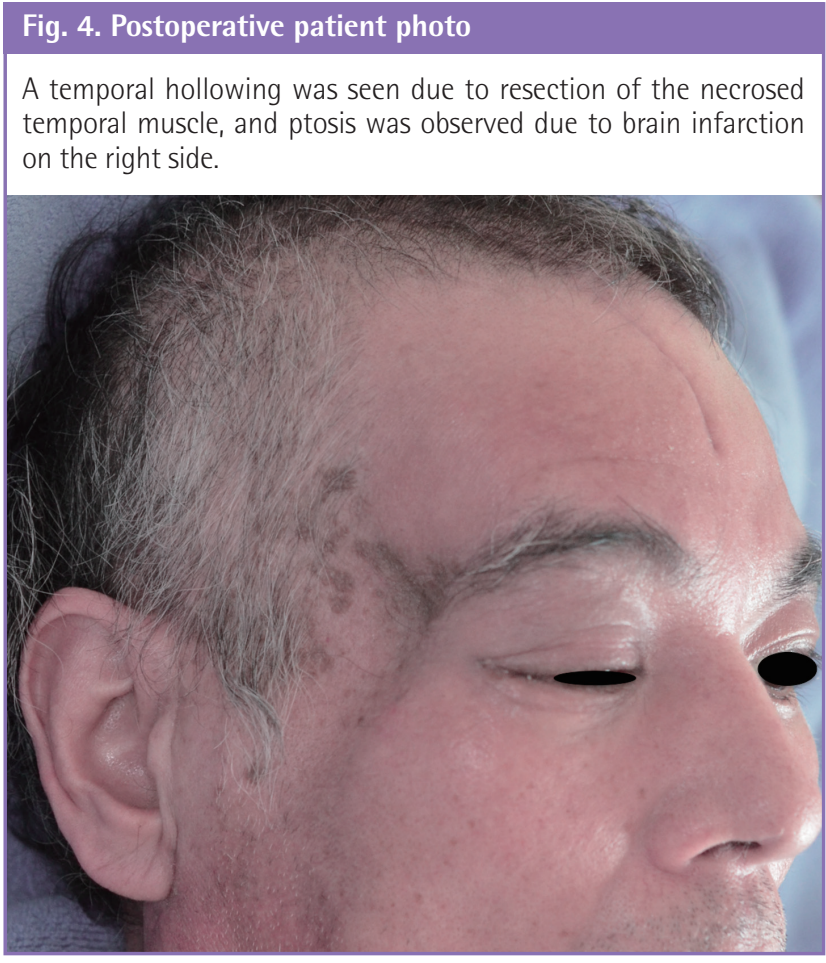

ing either the lateral orbital approach or the cranial approach. The lateral orbital approach can provide direct access to the intraorbital region, but the surgical field is too narrow and deep to reach the abscess cavity properly. In other circumstances, the lateral orbital approach can be combined with a trans-nasal endoscopic approach to manipulate the deep orbital area. However, in this case, we did not have an otolaryngologist who could provide a trans-nasal endoscopic approach. Therefore, our approach might be useful when it is not possible to request a trans- 
nasal assist. The cranial approach can also provide wide exposure of the intraorbital region, but this approach requires removal of the orbital roof, which results in the possibility of linkage between the intraorbital abscess and the intracranial region. This communication might induce contamination of the intracranial region via the intraorbital abscess, potentially causing a life-threatening condition such as an epidural abscess. Moreover, for a multifocal abscess in the craniofacial region, both approaches require multiple additional incisions to the face.

The advantages of the FDF approach are its wide and clear surgical field from the cranial region to the mid-face and the fact that it leaves no unpleasant scars on the face and has no functional complications. Moreover, the FDF approach provides easy access to the lateral orbital area, which makes it easier to use the lateral orbital approach. If the FDF approach is combined with the lateral orbital approach, surgeons will have enough space to reach and work on an abscess cavity in the intraorbital region, with less risk of damaging the optic nerve and/ or orbital vessels.

\section{NOTES}

\section{Conflict of interest}

No potential conflict of interest relevant to this article was reported.

\section{Patient consent}

The patient provided written informed consent for the publication and the use of his images.

\section{REFERENCES}

1. Tajima S, Tanaka Y, Imai K, et al. Extended coronal flap"Dismasking flap" for craniofacial and skull base surgery. Bull Osaka Med Coll 1993;39:1-8.

2. Koda H, Tsunoda A, Iida H, et al. Facial dismasking flap for removal of tumors in the craniofacial region. Laryngoscope 2007;117:1533-8.

3. Kishimoto S, Tsunoda A, Koda H. Nasal downward swing approach coupled with the facial dismasking flap. Auris Nasus Larynx 2010;37:217-9.

4. Fujimoto T, Imai K, Takahashi M, et al. Retrospective assessment of the dismasking flap procedure as a craniofacial approach. J Neurosurg Pediatr 2011;7:345-50.

5. Yano T, Okazaki M, Tanaka K, et al. indication for and limitation of the facial dismasking flap approach for skull base surgery to achieve the best esthetical and functional results. Ann Plast Surg 2017;78:49-53. 